Discrete Comput Geom 29:83-104 (2003)

DOI: $10.1007 / \mathrm{s} 00454-002-2811-7$

Geometry

\title{
Circular Cylinders through Four or Five Points in Space*
}

\author{
Olivier Devillers, ${ }^{1}$ Bernard Mourrain, ${ }^{1}$ Franco P. Preparata, ${ }^{2}$ and Philippe Trebuchet ${ }^{1}$ \\ ${ }^{1}$ INRIA, Unité de Recherche Sophia Antipolis, \\ BP93, 06902 Sophia-Antipolis, France \\ \{Olivier.Devillers,Bernard.Mourrain,Philippe.Trebuchet\}@ sophia.inria.fr \\ ${ }^{2}$ Center for Geometric Computing, Department of Computer Science, Brown University, \\ 115 Waterman Street, Providence, RI 02912, USA \\ franco@cs.brown.edu
}

\section{Introduction}

The focus of this paper is the analysis of circular cylinders through sets of points in three dimensions. This investigation has a number of motivations. Clearly, if a cylinder of radius $R$ and direction $\mathbf{t}$ passes through a set of points $P$, it means also that there is a line of direction $\mathbf{t}$ tangent to the sphere of radius $R$ and centered at the points of $P$. Another interpretation is that an observer looking at $P$ from infinity in direction $\mathbf{t}$ sees the points of $P$ as cocircular. So, this kind of operations arises in surface reconstruction, visibility and metrology.

In Section 2 we describe precisely the set of cylinders through the vertices of a regular tetrahedron. These preliminary observations give insights into the problem and provide lower bounds on the number of solutions. In Section 3, given four points in space, we prove a degree three condition on a direction of a cylinder through these four points. We exploit this result to prove that there are at most six cylinders through five points (Section 4), at most 186 cylindrical shells through six points (Section 5), and at most nine pairs of parallel cylinders through two sets of four points (Section 6). These bounds are tight as illustrated by explicit examples. Such primitives are used for smallest enclosing cylinders [1], [17] or for Delaunay triangulation of projected points [4]. The number of cylindrical shells appear in metrology problems [2], [8].

The first problem of cylinders through five points has already been considered in the literature. The problem of cylinders through four and five points is discussed by Bottema

\footnotetext{
* This work was partially supported by the U.S. Army Research Office under Grant DAAH04-96-1-0013. Part of this work was done while O. Devillers was visiting Brown University.
} 
and VeldKamp [5], using "complicated" line geometry; in particular, they gave a bound on the number of cylinders through five points. This problem has been reinvestigated by Lichtblau [11] using calculus with a computer algebra system.

Searching for cylinders of some fixed radius can be interesting for collision detection, visibility or the existence of enclosing cylinders with a given radius. We investigate this problem in Section 7 and prove a tight bound of 12 solutions. The same bound was recently obtained by Macdonald et al. [12] using a different approach, and its tightness is established also using the regular tetrahedron.

The smallest (or largest) cylinder enclosing (or "surrounded by") a set of points can be defined by five or fewer points, so that it is of interest to study extremal-radius cylinders through four points. We prove in Section 8 that their number is at most 18.

\section{The Views of Regular Tetrahedra}

\subsection{A Single Regular Tetrahedron}

In this section we investigate the different views of the four vertices of a regular tetrahedron. More precisely, given four points $p_{1}=(0,0,0), p_{2}=(1,0,0), p_{3}=$ $(1 / 2, \sqrt{3} / 2,0)$ and $p_{4}=(1 / 2, \sqrt{3} / 6, \sqrt{6} / 3)$, we will project these four points on a plane orthogonal to the direction $\mathbf{t}$ and look at the Delaunay triangulation of the four points. By definition, the direction $\mathbf{t}$ is an element of the two-dimensional projective plane $\mathbb{P}^{2}$. It will be represented by three coordinates $\mathbf{t}=(l: m: n)$ with $(l, m, n) \neq \mathbf{0}$ and $(l: m: n) \equiv \lambda(l: m: n)$ for $\lambda \neq 0$. Conveniently, $\mathbf{t}$ can also be viewed as a unit vector, and we identify $\mathbf{t}$ with $-\mathbf{t}$. We draw a diagram $\mathcal{D}$ which partitions the sphere of directions into maximal connected regions so that the Delaunay triangulations of the projection of the points for all directions within each region are the same. Since $\mathbf{t}$ and $-\mathbf{t}$ define the same direction, only half of the sphere is relevant. Thus we are seeking directions where the topology of the Delaunay triangulation changes.

Clearly, if $\mathbf{t}$ is parallel to a face of the tetrahedron, then three points are collinear in projection. In such a case, $\mathbf{t}$ describes a point of an edge of $\mathcal{D}$, separating a region where the convex hull of the projected point is a triangle from the one in which it is a quadrilateral; these directions yield four great circles of the unit sphere (belonging to $\mathcal{D})$, drawn in dashed lines in Fig. 1.

If $\mathbf{t}$ is parallel to the plane generated by two opposite edges of the tetrahedron, the four projected points form a trapezoid with an axis of symmetry, so that the four points are clearly cocircular; thus we get three other great circles, drawn in grey in Fig. 1, belonging to $\mathcal{D}$. We point out that for four points in general position, the grey curve no longer consists of a set of great circles, but, as we will see in Section 3, it will be a cubic curve.

Figure 1 shows the view of $\mathcal{D}$ from $z=+\infty$ in direction $(0,0,-1)$. Grey curves correspond to cocircularity directions and dashed curves to collinearity directions. The intersections between dashed curves correspond to the six directions of the edges of the tetrahedron. In such a case, two vertices project at the same point, so that the Delaunay triangulation is a single triangle, and thus the four points are cocircular. It follows that each of these directions belongs to a branch of the grey curve. These points are marked by a triangle on Fig. 1. Figure 2 is the central projection of Fig. 1 on a generic plane. 

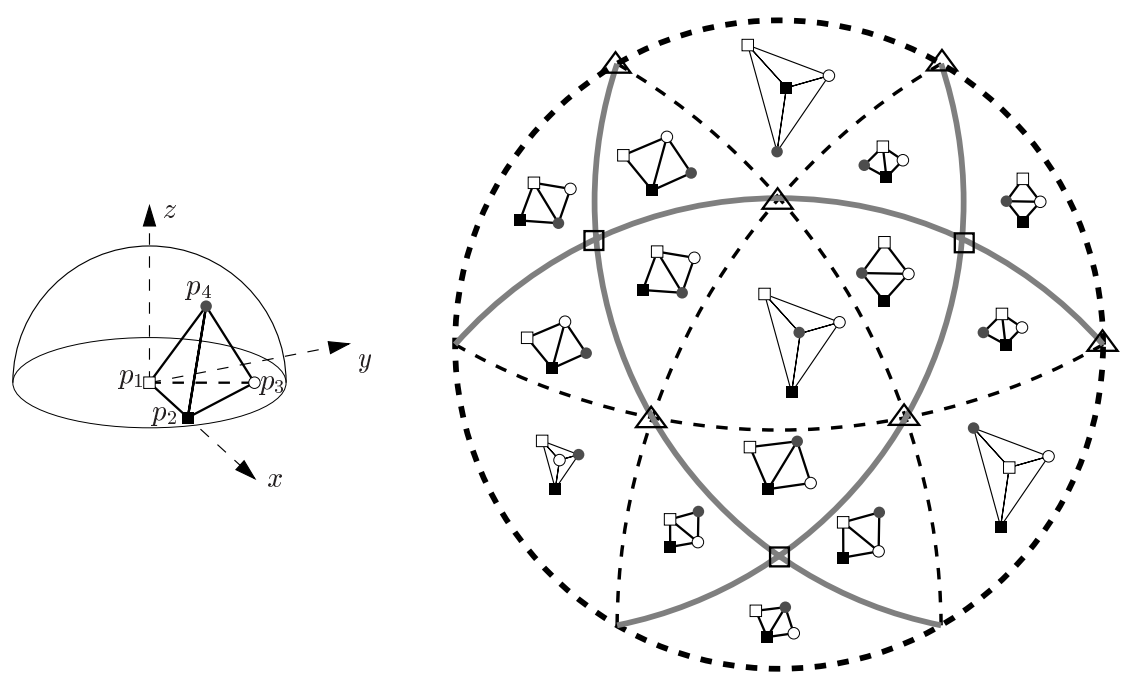

Fig. 1. Different views of fours points.

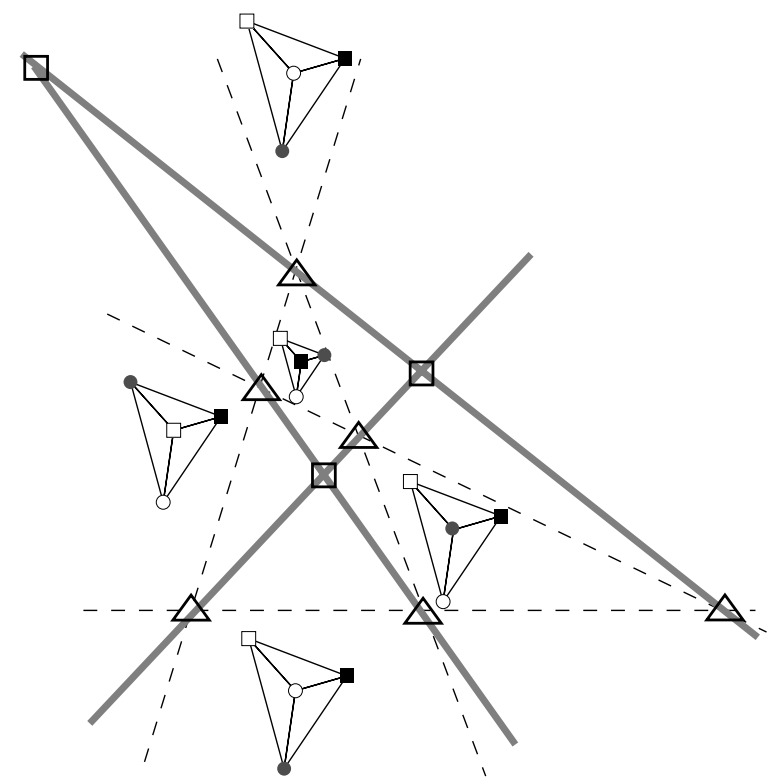

Fig. 2. The same picture in the projective plane. 
At the intersection of two branches of the grey curve, the projection of the four points is a square, and these three directions are marked by a square. When the direction of projection moves from a triangle-direction to a square-direction on the grey curve the radius of the circle circumscribing the four points is decreasing, so that the circle passing through the four points has minimal radius $\left(\frac{1}{2}=0.5\right)$ at the three square-directions, maximal radius $(3 \sqrt{2} / 8 \simeq 0.53)$ at the six triangle-directions and any given radius inbetween in at least 12 different points (one on each grey edge between a triangle-point and a square-point). This will be confirmed by the algebraic developments of Section 7 . In Section 8 we analyze in detail the multiplicity of these extremal points.

\subsection{Two Regular Tetrahedra Sharing a Face}

Referring to Fig. 3 , if we add the point $p_{5}=(1 / 2,-\sqrt{3} / 6,-\sqrt{6} / 3)$ (crossed disk) and consider the two tetrahedra $\left(p_{1}, p_{2}, p_{3}, p_{4}\right)$ and $\left(p_{1}, p_{2}, p_{3}, p_{5}\right)$, we can draw on the sphere the two corresponding diagrams. Due to the symmetry of the configuration, the diagram of $\left(p_{1}, p_{2}, p_{3}, p_{5}\right)$ is obtained from the diagram of $\left(p_{1}, p_{2}, p_{3}, p_{4}\right)$ by a rotation of $\pi$.

In Fig. 3 collinearity curves are shown short-dashed for $\left(p_{1}, p_{2}, p_{3}, p_{4}\right)$ and longdashed for $\left(p_{1}, p_{2}, p_{3}, p_{5}\right)$, cocircularity curves are in grey for $\left(p_{1}, p_{2}, p_{3}, p_{4}\right)$ and in light grey for $\left(p_{1}, p_{2}, p_{3}, p_{5}\right)$. There are six directions along which the five points have cocircular projections, marked by a circle on the figure, at the intersection of grey and light grey cocircularity curves. There are also three directions where two of the points $\left(p_{1}, p_{2}, p_{3},\right)$ project on the same point, and thus $\left(p_{1}, p_{2}, p_{3}, p_{5}\right)$ and $\left(p_{1}, p_{2}, p_{3}, p_{4}\right)$ are cocircular but on different circles; these points are marked by a pair of intersecting circles in the figure.
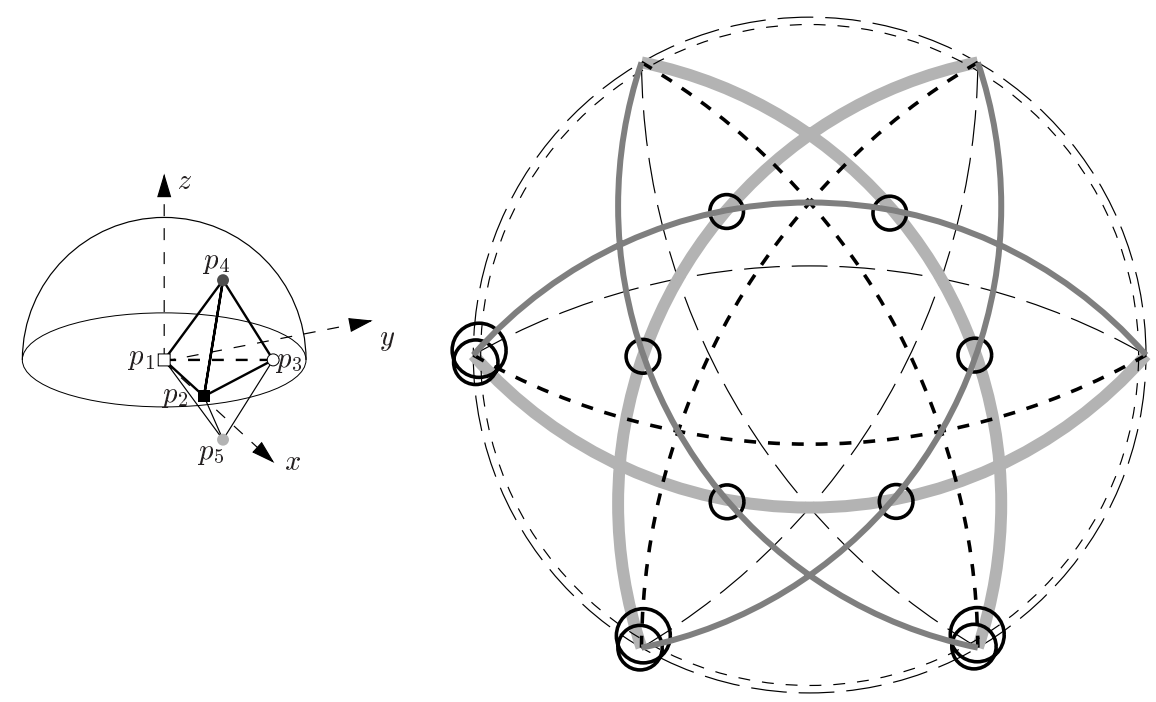

Fig. 3. There are six cylinders through five points. 

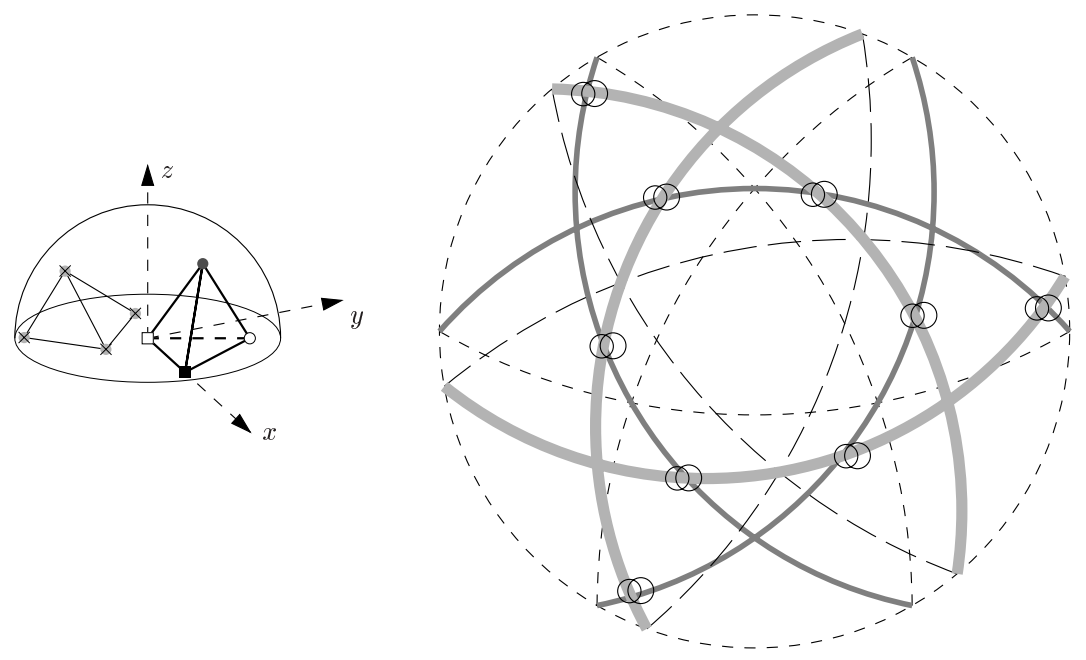

Fig. 4. There are nine pairs of parallel cylinders through two sets of four points.

Thus for the six directions where the five points project cocircularly, there is a cylinder with that direction passing through the five points.

\subsection{Two Disjoint Regular Tetrahedra}

Given two sets of four points, we are interested in the directions along which both sets have cocircular projections. As noted in Section 2.1 the set of directions along which four points have cocircular projections is defined by three great circles on the sphere. Thus given two regular tetrahedra we get nine directions belonging to the corresponding two sets of three great circles.

Figure 4 describes a case where both regular tetrahedra have a horizontal face, so that the diagram for the second tetrahedron is just a rotation of the first one. Each of the nine directions is marked by two small circles on the figure.

\section{Cylinders through Four Points}

With the intuition gained from the preceding geometric considerations, we can now move to an algebraic analysis. We begin by considering the equations defining the set of cylinders passing through four points in the space. Given four points $p_{1}, p_{2}, p_{3}, p_{4}$ in three-dimensional space, we wish to analyze the set of directions corresponding to cylinders passing through these points. Without loss of generality, modulo a rigid motion, we may assume that $p_{1}=(0,0,0), p_{2}=\left(x_{2}, 0,0\right), p_{3}=\left(x_{3}, y_{3}, 0\right)$ and $p_{4}=\left(x_{4}, y_{4}, z_{4}\right)$.

Let $\mathbf{t}=(l, m, n)$ be the unit vector identifying a direction $(\mathbf{t} \equiv(l: m: n)$ in $\mathbb{P}^{2}$ ). Consider now the plane $\pi$ through the origin and orthogonal to $\mathbf{t}$, and a system of 
coordinates $(X, Y, Z)$ having its two first axes in $\pi$ and the third axis of direction $\mathbf{t}$. The transformation of coordinates from $(x, y, z)$ to $(X, Y, Z)$ brings the $Z$-axis to coincide with the direction $\mathbf{t}$. Among all possible transformations (corresponding to an arbitrary rotation around the $Z$-axis) we select the one specified by the following unitary matrix, where $\rho^{2}=m^{2}+n^{2}$ :

$$
M=\left(\begin{array}{ccc}
\rho & -\frac{l m}{\rho} & -\frac{n l}{\rho} \\
0 & \frac{n}{\rho} & -\frac{m}{\rho} \\
l & m & n
\end{array}\right)
$$

Let $\left(X_{i}, Y_{i}, Z_{i}\right)$ be the coordinates of $p_{i}$ in system $(X, Y, Z)$. The orthogonal projection $q_{i}$ of $p_{i}$ on $\pi$ in system $(X, Y, Z)$ in terms of $\left(x_{i}, y_{i}, z_{i}\right)$ has coordinates

$$
\left(\rho x_{i}-\frac{l m}{\rho} y_{i}-\frac{n l}{\rho} z_{i}, \frac{n}{\rho} y_{i}-\frac{m}{\rho} z_{i}, 0\right),
$$

where the last coordinates is 0 as expected.

The points $p_{1}, p_{2}, p_{3}, p_{4}$ belong to a cylinder of direction $\mathbf{t}$ if and only if the points $q_{1}, q_{2}, q_{3}, q_{4}$ are cocircular in $\pi$, that is,

$$
\mathcal{C}_{p_{1}, p_{2}, p_{3}, p_{4}}(l, m, n)=\left|\begin{array}{cccc}
1 & 1 & 1 & 1 \\
X_{1} & X_{2} & X_{3} & X_{4} \\
Y_{1} & Y_{2} & Y_{3} & Y_{4} \\
X_{1}^{2}+Y_{1}^{2} & X_{2}^{2}+Y_{2}^{2} & X_{3}^{2}+Y_{3}^{2} & X_{4}^{2}+Y_{4}^{2}
\end{array}\right|=0 .
$$

For points $p_{1}$ and $p_{2},(2)$ gives the coordinates of $q_{1}$ and $q_{2}$ :

$$
X_{1}=Y_{1}=Z_{1}=0 ; \quad X_{2}=\rho x_{2} ; \quad Y_{2}=Z_{2}=0 .
$$

Since $q_{i}(i=3,4)$ is orthogonal to the unit vector $\mathbf{t}$, we obtain

$$
X_{i}^{2}+Y_{i}^{2}=\left|q_{i}\right|^{2}=\left|p_{i}\right|^{2}-\left(\mathbf{t} \cdot p_{i}\right)^{2} .
$$

Developing determinant $\mathcal{C}_{p_{1}, p_{2}, p_{3}, p_{4}}(l, m, n)$ we get

$$
\begin{aligned}
\mathcal{C}_{p_{1}, p_{2}, p_{3}, p_{4}}(l, m, n) & \\
& =\left|\begin{array}{cccc}
1 & 1 & 1 & 1 \\
0 & \rho x_{2} & \rho x_{3}-\frac{l m}{\rho} y_{3} & \rho x_{4}-\frac{l m}{\rho} y_{4}-\frac{n l}{\rho} z_{4} \\
0 & 0 & \frac{n}{\rho} y_{3} & \frac{n}{\rho} y_{4}-\frac{m}{\rho} z_{4} \\
0 & \rho^{2} x_{2}^{2} & \left|p_{3}\right|^{2}-\left(\mathbf{t} \cdot p_{3}\right)^{2} & \left|p_{4}\right|^{2}-\left(\mathbf{t} \cdot p_{4}\right)^{2}
\end{array}\right| \\
& =\rho x_{2}\left|\begin{array}{ccc}
\frac{n}{\rho} y_{3} & \frac{n}{\rho} y_{4}-\frac{m}{\rho} z_{4} \\
\left|p_{3}\right|^{2}-\left(\mathbf{t} \cdot p_{3}\right)^{2} & \left|p_{4}\right|^{2}-\left(\mathbf{t} \cdot p_{4}\right)^{2}
\end{array}\right|
\end{aligned}
$$




$$
+\rho^{2} x_{2}^{2}\left|\begin{array}{cc}
\rho x_{3}-\frac{l m}{\rho} y_{3} & \rho x_{4}-\frac{l m}{\rho} y_{4}-\frac{n l}{\rho} z_{4} \\
\frac{n}{\rho} y_{3} & \frac{n}{\rho} y_{4}-\frac{m}{\rho} z_{4}
\end{array}\right| .
$$

The first term can be rewritten as

$$
\begin{aligned}
& x_{2}\left|\begin{array}{cc}
n y_{3} & n y_{4}-m z_{4} \\
\left|p_{3}\right|^{2}-\left(\mathbf{t} \cdot p_{3}\right)^{2} & \left|p_{4}\right|^{2}-\left(\mathbf{t} \cdot p_{4}\right)^{2}
\end{array}\right| \\
& =-x_{2}\left|\begin{array}{cc}
\left|\begin{array}{cc}
m & y_{3} \\
n & 0
\end{array}\right| & \left|\begin{array}{cc}
m & y_{4} \\
n & z_{4}
\end{array}\right| \\
\left|p_{3}\right|^{2}-\left(\mathbf{t} \cdot p_{3}\right)^{2} & \left|p_{4}\right|^{2}-\left(\mathbf{t} \cdot p_{4}\right)^{2}
\end{array}\right| \\
& =-x_{2}\left|\begin{array}{ccc}
m & y_{3} & y_{4} \\
n & 0 & z_{4} \\
0 & \left|p_{3}\right|^{2}-\left(\mathbf{t} \cdot p_{3}\right)^{2} & \left|p_{4}\right|^{2}-\left(\mathbf{t} \cdot p_{4}\right)^{2}
\end{array}\right| \\
& =-x_{2}\left|\begin{array}{ccc}
m & y_{3} & y_{4} \\
n & 0 & z_{4} \\
0 & (\mathbf{t} \cdot \mathbf{t})\left|p_{3}\right|^{2}-\left(\mathbf{t} \cdot p_{3}\right)^{2} & (\mathbf{t} \cdot \mathbf{t})\left|p_{4}\right|^{2}-\left(\mathbf{t} \cdot p_{4}\right)^{2}
\end{array}\right| .
\end{aligned}
$$

The second term is

$$
\begin{aligned}
\rho^{2} x_{2}^{2}\left(n x_{3} y_{4}-\right. & \left.x_{3} m z_{4}-\frac{l m n}{\rho^{2}} y_{3} y_{4}+\frac{l m^{2}}{\rho^{2}} y_{3} z_{4}\right)-\rho^{2} x_{2}^{2}\left(n x_{4} y_{3}-\frac{l m n}{\rho^{2}} y_{3} y_{4}-\frac{l n^{2}}{\rho^{2}} y_{3} z_{4}\right) \\
= & x_{2}^{2}\left(n^{2}+m^{2}\right)\left(n x_{3} y_{4}-m x_{3} z_{4}-n x_{4} y_{3}+l y_{3} z_{4}\right) \\
& \quad\left(\text { because } \rho^{2}=n^{2}+m^{2}\right) \\
= & x_{2}^{2}\left(n^{2}+m^{2}\right)\left|\begin{array}{ccc}
l & x_{3} & x_{4} \\
m & y_{3} & y_{4} \\
n & 0 & z_{4}
\end{array}\right| .
\end{aligned}
$$

We conclude that

$$
\begin{aligned}
\mathcal{C}_{p_{1}, p_{2}, p_{3}, p_{4}}(l, m, n)= & x_{2}^{2}\left(m^{2}+n^{2}\right)\left|\begin{array}{ccc}
l & x_{3} & x_{4} \\
m & y_{3} & y_{4} \\
n & 0 & z_{4}
\end{array}\right| \\
& -x_{2}\left|\begin{array}{ccc}
m & y_{3} & y_{4} \\
n & 0 & z_{4} \\
0 & (\mathbf{t} \cdot \mathbf{t})\left|p_{3}\right|^{2}-\left(\mathbf{t} \cdot p_{3}\right)^{2} & (\mathbf{t} \cdot \mathbf{t})\left|p_{4}\right|^{2}-\left(\mathbf{t} \cdot p_{4}\right)^{2}
\end{array}\right| .
\end{aligned}
$$

The preceding analysis, starting from the definition of matrix $M$ and ending with the above expression of $\mathcal{C}_{p_{1}, p_{2}, p_{3}, p_{4}}(l, m, n)$ rests on the identity $l^{2}+m^{2}+n^{2}=1$. Thus if we view $\mathcal{C}_{p_{1}, p_{2}, p_{3}, p_{4}}(l, m, n)=0$ as the equation of a surface in three-dimensional space, we are actually considering its intersection with the unit sphere of equation $l^{2}+m^{2}+n^{2}=1$. Alternatively, we may view $(l, m, n)$ as a point of the projective space $\mathbb{P}^{2}$ of nonzero 
directions, and we conclude that the set of directions for which $p_{1}, p_{2}, p_{3}$ and $p_{4}$ are cocircular verifies $\mathcal{C}_{p_{1}, p_{2}, p_{3}, p_{4}}(l, m, n)=0$, which describes a curve of the projective space $\mathbb{P}^{2}$ of nonzero directions. Hereafter, we deal with homogeneous polynomials, in order to apply Bézout's theorem, which yields the exact number of roots (counted with multiplicities) for proper intersections of curves in the projective plane.

When we choose for $(l, m, n)$ one of the directions $\left(p_{i}, p_{j}\right), 1 \leq i \neq j \leq 4$, points $p_{i}$ and $p_{j}$ project to the same point in $\pi$ and correspondingly polynomial $\mathcal{C}_{p_{1}, p_{2}, p_{3}, p_{4}}$ vanishes. Thus we have the following result.

Theorem 1. The directions such that four points are cocircular when projecting onto a plane perpendicular to that direction belong to a curve of degree three of the projective space $\mathbb{P}^{2}$, passing by the six directions $\left(p_{i}, p_{j}\right)$ for $1 \leq i \neq j \leq 4$.

\section{Cylinders through Five Points}

Let $p_{1}, p_{2}, p_{3}, p_{4}$ and $p_{5}$ be five distinct points in three-dimensional space. We are seeking the direction along which the five points belong to the same cylinder. Necessarily, such a direction $(l, m, n)$ must verify the following conditions:

$$
\begin{aligned}
& \mathcal{C}_{p_{1}, p_{2}, p_{3}, p_{4}}(l, m, n)=0, \\
& \mathcal{C}_{p_{1}, p_{2}, p_{3}, p_{5}}(l, m, n)=0 .
\end{aligned}
$$

More specifically:

Theorem 2. The number of circular cylinders through five points is at most six. This bound on the number of real solutions is attained by some configurations.

Proof. Since both equations in (5) are of degree three, by Bézout's theorem the number of points common to the two curves (when finite) is bounded by nine. However, the three directions $p_{1} p_{2}, p_{1} p_{3}$ and $p_{2} p_{3}$ although solutions of the system, do not correspond to directions where the five points are cocircular. Indeed, they identify pairs of cylinders sharing one generatrix.

Removal of these three solutions leaves a maximum number of six solutions.

This bound is tight as shown by the example of two regular tetrahedra sharing a face given in Section 2.2, for which there are six feasible directions listed below:

\begin{tabular}{ccc}
\hline$l$ & $m$ & $n$ \\
\hline $1 / \sqrt{10}$ & $1 / 10 \sqrt{2} \sqrt{15}$ & $1 / 5 \sqrt{15}$ \\
$1 / \sqrt{10}$ & $1 / 10 \sqrt{2} \sqrt{15}$ & $-1 / 5 \sqrt{15}$ \\
$1 / \sqrt{10}$ & $-1 / 10 \sqrt{2} \sqrt{15}$ & $1 / 5 \sqrt{15}$ \\
$1 / \sqrt{10}$ & $-1 / 10 \sqrt{2} \sqrt{15}$ & $-1 / 5 \sqrt{15}$ \\
$2 / \sqrt{10}$ & 0 & $1 / 5 \sqrt{15}$ \\
$2 / \sqrt{10}$ & 0 & $-1 / 5 \sqrt{15}$ \\
\hline
\end{tabular}


Note that this bound is not attained in general if we restrict ourselves to real solutions. To illustrate this remark, it suffices to take as the set of points the vertices of a tetrahedron and a fifth point inside that tetrahedron; in this case, obviously there is no (real) cylinder passing through the five points and the six solutions of our system are all with complex coordinates.

If we count, however, the number of complex roots with their multiplicities (which we may have to consider for instance if we are in the neighborhood of a singular configuration), this bound is exact for almost all set of five points, according to Bézout's theorem.

\section{Cylindrical Shells through Six Points}

For metrology applications, we are interested in zone cylinders or cylindrical shells which are in fact pairs of coaxial cylinders. We call the positive difference of their radii the width of the zone cylinder. Finding a minimal-width zone cylinder containing a set of points $P$ is an important problem in metrology. Such a cylinder passes through (is defined by) six points of $P$. The partitions of the defining points $p_{1}, p_{2}, p_{3}, p_{4}, p_{5}$ and $p_{6}$ between the internal and external cylinder may be of the following types:

$-\left\{p_{1}, p_{2}, p_{3}, p_{4}, p_{5}\right\},\left\{p_{6}\right\}$ corresponding to the following (cocircularity) conditions:

$$
\begin{aligned}
& \mathcal{C}_{p_{1}, p_{2}, p_{3}, p_{4}}(l, m, n)=0, \\
& \mathcal{C}_{p_{1}, p_{2}, p_{3}, p_{5}}(l, m, n)=0 .
\end{aligned}
$$

$-\left\{p_{1}, p_{2}, p_{3}, p_{4}\right\},\left\{p_{5}, p_{6}\right\}$ corresponding to the following conditions:

$$
\begin{aligned}
& \mathcal{C}_{p_{1}, p_{2}, p_{3}, p_{4}}(l, m, n)=0, \\
& \mathcal{C}_{p_{1}, p_{2}, p_{3}, p_{5}}(l, m, n)=\mathcal{C}_{p_{1}, p_{2}, p_{3}, p_{6}}(l, m, n),
\end{aligned}
$$

where the latter specifies that $p_{5}$ and $p_{6}$ are equidistant from the axis.

$-\left\{p_{1}, p_{2}, p_{3}\right\},\left\{p_{4}, p_{5}, p_{6}\right\}$ corresponding to the conditions:

$$
\begin{aligned}
& \mathcal{C}_{p_{1}, p_{2}, p_{3}, p_{4}}(l, m, n)=\mathcal{C}_{p_{1}, p_{2}, p_{3}, p_{5}}(l, m, n), \\
& \mathcal{C}_{p_{1}, p_{2}, p_{3}, p_{4}}(l, m, n)=\mathcal{C}_{p_{1}, p_{2}, p_{3}, p_{6}}(l, m, n),
\end{aligned}
$$

where both conditions specify that $\left\{p_{4}, p_{5}, p_{6}\right\}$ are equidistant from the axis.

In all three cases we get a system of two equations of degree three, and in all cases $p_{1} p_{2}, p_{1} p_{3}$ and $p_{2} p_{3}$ are irrelevant solutions, so that in all cases there are at most six solutions for any given partition. Partitions of the first, second and third types are selectable in six, fifteen and ten ways, respectively, and we have the following theorem:

Theorem 3. The number of cylindrical shells through six points is at most 186.

If we are interested in shells of locally minimum width, each cylinder (internal or external) must pass through at least two points [2], [8], thus reducing the number of possible partitions of the six points, and the bound on the number of shells to 150 . 


\section{Pair of Parallel Cylinders through Two Sets of Four Points}

Given two sets of points, $p_{1}, p_{2}, p_{3}, p_{4}$ and $p_{1}^{\prime}, p_{2}^{\prime}, p_{3}^{\prime}, p_{4}^{\prime}$ in three-dimensional space, we are seeking directions along which both sets of points have cocircular projections, or, in other words, directions such that there exists a pair of parallel cylinders in that direction, each of them passing through a set of four points.

Theorem 4. The number of pairs of parallel circular cylinders through two sets of four points is at most nine. This bound on the number of real solutions is attained by some configurations.

Proof. Clearly the searched directions satisfy the two cubic equations:

$$
\begin{aligned}
& \mathcal{C}_{p_{1}, p_{2}, p_{3}, p_{4}}(l, m, n)=0, \\
& \mathcal{C}_{p_{1}^{\prime}, p_{2}^{\prime}, p_{3}^{\prime}, p_{4}^{\prime}}(l, m, n)=0 .
\end{aligned}
$$

This system has nine solutions in $\mathbb{P}^{2}$ by Bézout's theorem. This bound is tight as shown by the example of two regular tetrahedra given in Section 2.3 where the number of different directions attain the bound of nine.

\section{Cylinders through Four Points of a Given Radius}

We consider now the problem of cylinders with fixed radius through four points, which occurs in collision detection or visibility tests.

Theorem 5. The number of circular cylinders of given radius through four points in general position is at most 12 . This bound on the number of real solutions is attained in some configurations.

Proof. The cylinders of radius $\rho$ through four points $p_{1}, \ldots, p_{4}$ are determined by the two equations

$$
\begin{aligned}
\mathcal{C}_{p_{1}, p_{2}, p_{3}, p_{4}}(l, m, n) & =0, \\
\operatorname{radius}_{p_{1}, p_{2}, p_{3}}(l, m, n) & =\rho,
\end{aligned}
$$

where $\operatorname{radius}_{p_{1}, p_{2}, p_{3}}(l, m, n)$ is the radius of the circle defined by the projections $q_{1}, q_{2}, q_{3}$ of points $p_{1}, p_{2}, p_{3}$ on a plane normal to direction $(l, m, n)$. It is well known that the radius $R$ of a circle through points $(A, B, C)$ in the plane is given by the formula

$$
\Gamma=R^{2}=\frac{d(A, B)^{2} d(A, C)^{2} d(B, C)^{2}}{16(\operatorname{area}(A, B, C))^{2}},
$$

where $d(A, B)$ is the Euclidean distance between points $A$ and $B$ and area $(A, B, C)$ is the area of the triangle defined by these three points. In our case (that is, $p_{1}=(0,0,0)$, 
$\left.p_{2}=\left(x_{2}, 0,0\right), p_{3}=\left(x_{3}, y_{3}, 0\right)\right)$, we obtain, for the numerator,

$N(l, m, n)=\left(|\mathbf{t}|^{2}\left|p_{1} p_{2}\right|^{2}-\left(\mathbf{t} \cdot p_{1} p_{2}\right)^{2}\right)\left(|\mathbf{t}|^{2}\left|p_{1} p_{3}\right|^{2}-\left(\mathbf{t} \cdot p_{1} p_{3}\right)^{2}\right)\left(|\mathbf{t}|^{2}\left|p_{2} p_{3}\right|^{2}-\left(\mathbf{t} \cdot p_{2} p_{3}\right)^{2}\right)$

and, for the denominator,

$$
D(l, m, n)=4\left|\begin{array}{ccc}
1 & 1 & 1 \\
0 & \rho x_{2} & \rho x_{3}-\frac{l m}{\rho} y_{3} \\
0 & 0 & \frac{n}{\rho} y_{3}
\end{array}\right|^{2}|\mathbf{t}|^{4}=\left(2 n x_{2} y_{3}|\mathbf{t}|^{2}\right)^{2}
$$

Thus, system (7) consists of two homogeneous polynomials of degree respectively three and six. By Bézout's theorem, the number of projective roots counted with multiplicity is $3 \times 6=18$.

We now observe that each of the directions $p_{1} p_{2}, p_{1} p_{3}$ and $p_{2} p_{3}$ satisfies equation $\mathcal{C}_{p_{1}, p_{2}, p_{3}, p_{4}}(l, m, n)=0$ (with multiplicity one for non-collinear points). Moreover, we claim that each of these directions also satisfies equations $N(l, m, n)=0$ and $D(l, m, n)=0$. Indeed, setting $\mathbf{t}=p_{i} p_{j} /\left|p_{i} p_{j}\right|$ nullifies one of the three factors of $N(l, m, n)$ (referring to the expression of $N(l, m, n)$ given above, we note that this happens with multiplicity two). In addition, $\mathbf{t}=p_{i} p_{j}$ implies that $\mathbf{t}$ belongs to the plane of the triangle defined by points $p_{1}, p_{2}, p_{3}$, so that area $\left(q_{1}, q_{2}, q_{3}\right)=0$. In each of these cases the ratio $N / D$ is of the form $0 / 0$, so that these three degenerate solutions must be rejected and $\mathbf{t}=p_{i} p_{j}$ is a solution of multiplicity two of $N-\rho^{2} D=0$. Therefore, the number 18 of projective solutions of system (7) must be reduced by $3 \times 2=6$ (three solutions each of multiplicity two), yielding a bound of 12 .

This bound is tight as shown by the example of the regular tetrahedron given in Section 2.1. Indeed in Fig. 1 there are twelve segments between a square-point (corresponding to a minimum of the radius) and a triangle-point (corresponding to a maximum of the radius). Any value of the radius between the maximum and the minimum yields one real solution point on each of these curve segments.

Remark. It should not be construed, however, that a regular tetrahedron is the only configuration of points attaining the bound stated in the preceding theorem. In fact, it can be easily shown that the set of points $(0,0,0),(2,0,0),(1, a, 1),(1, a,-1)$, which gives a regular tetrahedron for $a=\sqrt{2}$, has a cocircularity curve consisting of three great circles, and each of them contains two maxima and two minima in each hemisphere.

\section{Cylinders of Extremal Radius through Four Points}

Theorem 6. The number of circular cylinders through four points with locally extremal radius is at most 18 .

Proof. Consider four points $p_{1}, \ldots, p_{4}$ in three-dimensional space. Again, and without loss of generality, we assume $p_{1}=(0,0,0), p_{2}=\left(x_{2}, 0,0\right)$ and $p_{3}=\left(x_{3}, y_{3}, 0\right)$. Next, 
we consider the set of cylinders through these four points of square radius $\Gamma$. They are defined by the following three equations:

$$
\begin{aligned}
\mathcal{C}_{p_{1}, p_{2}, p_{3}, p_{4}}(l, m, n) & =0, \\
\operatorname{radius}_{p_{1}, p_{2}, p_{3}}(l, m, n)^{2}-\Gamma & =0, \\
l^{2}+m^{2}+n^{2} & =1,
\end{aligned}
$$

which are homogeneous in the variables $(l, m, n, \Gamma)$. Using the condition $l^{2}+m^{2}+n^{2}=$ 1 , and the definitions of $N$ and $D$ in the previous section, the second of these equations can be rewritten as

$$
\begin{aligned}
\Gamma & =\frac{N(l, m, n)}{D(l, m, n)} \\
& =\frac{\left(|\mathbf{t}|^{2}\left|p_{1} p_{2}\right|^{2}-\left(\mathbf{t} \cdot p_{1} p_{2}\right)^{2}\right)\left(|\mathbf{t}|^{2}\left|p_{1} p_{3}\right|^{2}-\left(\mathbf{t} \cdot p_{1} p_{3}\right)^{2}\right)\left(|\mathbf{t}|^{2}\left|p_{2} p_{3}\right|^{2}-\left(\mathbf{t} \cdot p_{2} p_{3}\right)^{2}\right)}{4 y_{3}^{2} n^{2}},
\end{aligned}
$$

where, with our choices of points, $N(l, m, n)$ has degree six and $D(l, m, n)=D(n)$ has degree two.

Our task is to minimize or maximize $\Gamma$ subject to the constraints

$$
\begin{aligned}
\mathcal{C}_{p_{1}, p_{2}, p_{3}, p_{4}}(l, m, n) & =0, \\
l^{2}+m^{2}+n^{2} & =1 .
\end{aligned}
$$

Introducing Lagrange multipliers $\lambda_{1}$ and $\lambda_{2}$, this reduces to finding the extrema of the function

$$
\varphi=\frac{N(l, m, n)}{D(n)}+\lambda_{1} \mathcal{C}_{p_{1}, p_{2}, p_{3}, p_{4}}(l, m, n)+\lambda_{2}\left(l^{2}+m^{2}+n^{2}-1\right) .
$$

If we now set the partial derivatives of $\varphi$, with respect to $l, m$ and $n$, to zero, we can eliminate the multipliers $\lambda_{1}$ and $\lambda_{2}$ from the resulting three equations and obtain a single equation in $l, m$ and $n$. After some straightforward simplifications and using the abbreviated notation $\partial_{u}$ for $\partial / \partial u$ we obtain the equation

$$
\Delta:=\left|\begin{array}{ccc}
N \partial_{l} D-D \partial_{l} N & N \partial_{m} D-D \partial_{m} N & N \partial_{n} D-D \partial_{n} N \\
\partial_{l} C & \partial_{m} C & \partial_{n} C \\
l & m & n
\end{array}\right|=0
$$

to be paired with the equation

$$
C(l, m, n)=0 .
$$

Since $N, D$ and $C$ are of polynomial of degrees six, two, and three, respectively, (11) has degree ten. By Bézout's theorem, the number of joint solutions of system (11), (12) is $10 \times 3=30$.

As noted in the preceding section, however, the directions $p_{1} p_{2}, p_{1} p_{3}, p_{2} p_{3}$ are roots of the polynomials $N(l, m, n), D(n)$, with multiplicity two for each of them. Therefore, 
they are roots of the partial derivatives with multiplicity one; consequently, they are also roots of all terms of the first line of the determinant, as well as of (11) itself, with multiplicity at least three.

In fact, we use the following stronger result, which is proved after:

Proposition 7. In (11) the multiplicity of roots $p_{1} p_{2}, p_{1} p_{3}$, and $p_{2} p_{3}$ is at least four.

This result however does not extend to $p_{1} p_{4}, p_{2} p_{4}$ or $p_{3} p_{4}$, despite the equivalent roles that these directions play in our problem. In fact, in our analysis we have been considering directions of cocircularity for which the radius of the circle through $p_{1}, p_{2}$ and $p_{3}$ is extremal, thus attributing a different role to point $p_{4}$. The directions involving $p_{4}$ are roots of (12) but not of (11) (except in special configurations).

We now observe that $p_{1} p_{2}, p_{1} p_{3}$ and $p_{2} p_{3}$ with multiplicity four are improper solutions of our problem and should therefore be removed from the solution pool. ${ }^{1}$ In fact, for each of these directions the ratio $N / D$ is of the form $0 / 0$, so that there is always a cylinder passing also through $p_{4}$, with no implication of its having extremal radius. Thus, after removing these improper solutions, we conclude that the number of cylinders with extremal radius is at most $30-3 * 4=18$.

Proof of Proposition 7. We shall prove that $p_{1} p_{2}=\left(x_{2}, 0,0\right)$ is a root of (11) of multiplicity at least four. By analogy the result holds for $p_{1} p_{3}$ and $p_{2} p_{3}$ as well. We shall show that the Taylor expansion of the determinant at point $p_{1} p_{2}$ is expressible as a polynomial of valuation four, i.e., a polynomial all of whose monomials have degree at least four: this will establish the desired multiplicity of the root. To obtain the expansion at $p_{1} p_{2}$, let $\mathbf{t}^{\prime}=\left(l^{\prime}, m, n\right)=\mathbf{t}-p_{1} p_{2}$ and let $H_{i}$ be a generic polynomial in the variables $l^{\prime}, m, n$ of valuation $i$.

We now observe that for any vector $\mathbf{u}$,

$$
\begin{aligned}
|\mathbf{t}|^{2}|\mathbf{u}|^{2}-(\mathbf{t} \cdot \mathbf{u})^{2} & =\left(\left(\mathbf{t}^{\prime}+p_{1} p_{2}\right) \cdot\left(\mathbf{t}^{\prime}+p_{1} p_{2}\right)\right)|\mathbf{u}|^{2}-\left(\mathbf{t}^{\prime} \cdot \mathbf{u}+p_{1} p_{2} \cdot \mathbf{u}\right)^{2} \\
& =\left|p_{1} p_{2}\right|^{2}|\mathbf{u}|^{2}-\left(p_{1} p_{2} \cdot \mathbf{u}\right)^{2}+H_{1}
\end{aligned}
$$

which is specialized as

$$
\begin{aligned}
|\mathbf{t}|^{2}\left|p_{1} p_{2}\right|^{2}-\left(\mathbf{t} \cdot p_{1} p_{2}\right)^{2} & =\left(\left(x_{2}+l^{\prime}\right)^{2}+m^{2}+n^{2}\right) x_{2}^{2}-\left(\left(x_{2}+l^{\prime}\right) x_{2}\right)^{2}=\left(m^{2}+n^{2}\right) x_{2}^{2}, \\
|\mathbf{t}|^{2}\left|p_{1} p_{3}\right|^{2}-\left(\mathbf{t} \cdot p_{1} p_{3}\right)^{2} & =\left|p_{1} p_{2}\right|^{2}\left|p_{1} p_{3}\right|^{2}-\left(p_{1} p_{2} \cdot p_{1} p_{3}\right)^{2}+H_{1} \\
& =x_{2}^{2}\left(x_{3}^{2}+y_{3}^{2}\right)-\left(x_{2} x_{3}\right)^{2}+H_{1}=x_{2}^{2} y_{3}^{2}+H_{1}, \\
|\mathbf{t}|^{2}\left|p_{2} p_{3}\right|^{2}-\left(\mathbf{t} \cdot p_{2} p_{3}\right)^{2} & =\left|p_{1} p_{2}\right|^{2}\left|p_{2} p_{3}\right|^{2}-\left(p_{1} p_{2} \cdot p_{2} p_{3}\right)^{2}+H_{1} \\
& =x_{2}^{2}\left(\left(x_{3}-x_{2}\right)^{2}+y_{3}^{2}\right)-\left(x_{2}\left(x_{3}-x_{2}\right)\right)^{2}+H_{1}=x_{2}^{2} y_{3}^{2}+H_{1} .
\end{aligned}
$$

Therefore we obtain

$$
\begin{aligned}
N\left(x_{2}+l^{\prime}, m, n\right)= & \left(|\mathbf{t}|^{2}\left|p_{1} p_{2}\right|^{2}-\left(\mathbf{t} \cdot p_{1} p_{2}\right)^{2}\right)\left(|\mathbf{t}|^{2}\left|p_{1} p_{3}\right|^{2}-\left(\mathbf{t} \cdot p_{1} p_{3}\right)^{2}\right) \\
& \times\left(|\mathbf{t}|^{2}\left|p_{2} p_{3}\right|^{2}-\left(\mathbf{t} \cdot p_{2} p_{3}\right)^{2}\right)
\end{aligned}
$$

${ }^{1}$ This does not prevent any of the three directions from being a legitimate solution in special configurations. 


$$
\begin{aligned}
& =\left(m^{2}+n^{2}\right) x_{2}^{2}\left(x_{2}^{2} y_{3}^{2}+H_{1}\right)\left(x_{2}^{2} y_{3}^{2}+H_{1}\right) \\
& =x_{2}^{6} y_{3}^{4}\left(m^{2}+n^{2}\right)+H_{3} .
\end{aligned}
$$

The entries of the first line of the determinant can be rewritten as

$$
\begin{aligned}
N \partial_{l} D-D \partial_{l} N & =-D \partial_{l} N=H_{4}, \\
N \partial_{m} D-D \partial_{m} N & =-D \partial_{m} N=-4 y_{3}^{2} n^{2} x_{2}^{6} y_{3}^{4} 2 m+H_{4}=-8 x_{2}^{6} y_{3}^{6} m n^{2}+H_{4}, \\
N \partial_{n} D-D \partial_{n} N & =x_{2}^{6} y_{3}^{4}\left(m^{2}+n^{2}\right) 8 y_{3}^{2} n-4 y_{3}^{2} n^{2} x_{2}^{6} y_{3}^{4} 2 n+H_{4} \\
& =8 x_{2}^{6} y_{3}^{6} m^{2} n+H_{4}
\end{aligned}
$$

and correspondingly if we develop the determinant in (11) according to the first row and column we obtain

$$
\begin{gathered}
\left|\begin{array}{ccc}
H_{4} & -8 x_{2}^{6} y_{3}^{6} m n^{2}+H_{4} & 8 x_{2}^{6} y_{3}^{6} m^{2} n+H_{4} \\
\partial_{l} C & \partial_{m} C & \partial_{n} C \\
l^{\prime}+x_{2} & m & n
\end{array}\right| \\
=8 x_{2}^{6} y_{3}^{6}\left|\begin{array}{ccc}
0 & -m n^{2} & m^{2} n \\
0 & \partial_{m} C & \partial_{n} C \\
x_{2} & m & n
\end{array}\right|+H_{4} \partial_{l} C+H_{5} \\
=-8 x_{2}^{7} y_{3}^{6}\left(\partial_{m} C m+\partial_{n} C n\right) m n+H_{4} .
\end{gathered}
$$

We now claim that a polynomial $H_{2}$ of valuation two, can be chosen so that

$$
C_{p_{1}, p_{2}, p_{3}, p_{4}}\left(x_{2}+l^{\prime}, m, n\right)=\partial_{m} C m+\partial_{n} C n+H_{2} .
$$

Indeed, from the expression of $C$ in (5) we get

$$
\begin{aligned}
& C_{p_{1}, p_{2}, p_{3}, p_{4}}\left(x_{2}+l^{\prime}, m, n\right) \\
& =H_{2}-x_{2}\left|\begin{array}{ccc}
m & y_{3} & y_{4} \\
n & 0 & z_{4} \\
0 & x_{2}^{2} y_{3}^{2}+H_{1} & x_{2}^{2}\left(y_{4}^{2}+z_{4}^{2}\right)+H_{1}
\end{array}\right|=\lambda m+\mu n+H_{2}
\end{aligned}
$$

so that $\partial_{m} C m+\partial_{n} C n=\lambda m+\mu n+H_{2}$. We therefore conclude that the determinant in (11) can be expressed as

$$
\begin{array}{rl}
\Delta+8 x_{2}^{7} y_{3}^{6} & C(l, m, n) m n \\
= & -8 x_{2}^{7} y_{3}^{6}\left(\partial_{m} C m+\partial_{n} C n\right) m n+8 x_{2}^{7} y_{3}^{6}\left(\partial_{m} C m+\partial_{n} C n\right) m n+H_{4} \\
& =H_{4} .
\end{array}
$$

This proves that the multiplicity of $p_{1} p_{2}$ at the ideal $(D, C)=\left(D+8 x_{2}^{7} y_{3}^{6} C(l, m, n) m n\right.$, $C$ ) is at least four. By symmetry, this also holds for the other two directions.

Theorem 8. There exists a set of four points in three-dimensional space such that there are 18 circular cylinders through them counted with multiplicities. 
Proof. We report below the set of extremal cylinders obtained when the four points are the vertices of a regular tetrahedron. Solving the system of (11) and (12) for the points given in Fig. 1 yields nine distinct solutions counted with multiplicities (the sum of the multiplicity is 30 , that is, there is no complex solutions in this case). In the following table, $\mu$ is the multiplicity of the solution, $\rho$ is the corresponding radius and by $\mathbf{u} \times \mathbf{v}$ we denote, as customary, the external product of vector $\mathbf{u}$ and $\mathbf{v}$ :

\begin{tabular}{cccccc}
\hline $\mathbf{t}$ & $l$ & $m$ & $n$ & $\mu$ & $\rho$ \\
\hline$p_{1} p_{2}$ & 1 & 0 & 0 & 5 & $\frac{3}{8} \sqrt{2}$ \\
$p_{1} p_{3}$ & $\frac{1}{2}$ & $\sqrt{3} / 2$ & 0 & 5 & $\frac{3}{8} \sqrt{2}$ \\
$p_{2} p_{3}$ & $\frac{1}{2}$ & $-\sqrt{3} / 2$ & 0 & 5 & $\frac{3}{8} \sqrt{2}$ \\
$p_{1} p_{4}$ & $\frac{1}{2}$ & $\frac{1}{6} \sqrt{3}$ & $\frac{1}{3} \sqrt{6}$ & 1 & $\frac{3}{8} \sqrt{2}$ \\
$p_{2} p_{4}$ & $-\frac{1}{2}$ & $\frac{1}{6} \sqrt{3}$ & $\frac{1}{3} \sqrt{6}$ & 1 & $\frac{3}{8} \sqrt{2}$ \\
$p_{3} p_{4}$ & 0 & $-\frac{1}{3} \sqrt{3}$ & $\frac{1}{3} \sqrt{6}$ & 1 & $\frac{3}{8} \sqrt{2}$ \\
$p_{1} p_{2} \times p_{4} p_{3}$ & 0 & $\frac{1}{3} \sqrt{6}$ & $\frac{1}{3} \sqrt{3}$ & 4 & $\frac{1}{2}$ \\
$p_{1} p_{4} \times p_{3} p_{2}$ & $1 / \sqrt{2}$ & $1 / \sqrt{6}$ & $-1 / \sqrt{3}$ & 4 & $\frac{1}{2}$ \\
$p_{1} p_{3} \times p_{2} p_{4}$ & $1 / \sqrt{2}$ & $-1 / \sqrt{6}$ & $1 / \sqrt{3}$ & 4 & $\frac{1}{2}$ \\
\hline
\end{tabular}

In accordance with the preceding argument, directions $p_{1} p_{2}, p_{1} p_{3}$ and $p_{2} p_{3}$ are improper solutions of the system with multiplicity four; their removal (with multiplicity four) leaves each of them with multiplicity, thereby revealing their expected equivalence with directions $p_{1} p_{4}, p_{2} p_{4}$ and $p_{3} p_{4}$, so that the six edges correspond to solutions of extremal radius (maximal as observed in Section 2.1). On the other hand, directions $p_{1} p_{2} \times p_{4} p_{3}$, $p_{1} p_{4} \times p_{3} p_{2}$ and $p_{1} p_{3} \times p_{2} p_{4}$ (linking the midpoints of two opposite edges) are solutions of the system with multiplicity four and correspond to minima of the radius. In the example of the regular tetrahedron we get 18 real solutions counted with their multiplicity, matching the upper bound.

The previous configuration (a regular tetrahedron) is deformed by slightly perturbing the positions of the points $p_{1}, \ldots, p_{4}$ with the following results:

- The first three solution of multiplicity five give rise to a point (of multiplicity one) corresponding to a local maximum and to the irrelevant solutions $p_{1} p_{2}, p_{1} p_{3}, p_{2} p_{3}$, each with multiplicity four.

- The next three simple points remain simple and correspond to cylinders with locally maximal radius.

- Each point of the last set of three of multiplicity four is transformed into two distinct real minima and two additional complex points.

Equations (11) and (12) define two surfaces in three-dimensional space $l, m, n$. In Figs. 5 and 6 we present a cross section of these two surfaces with the plane $l=1$ in the case of the regular tetrahedron (Fig. 5) and the perturbed regular tetrahedron (Fig. 6). 


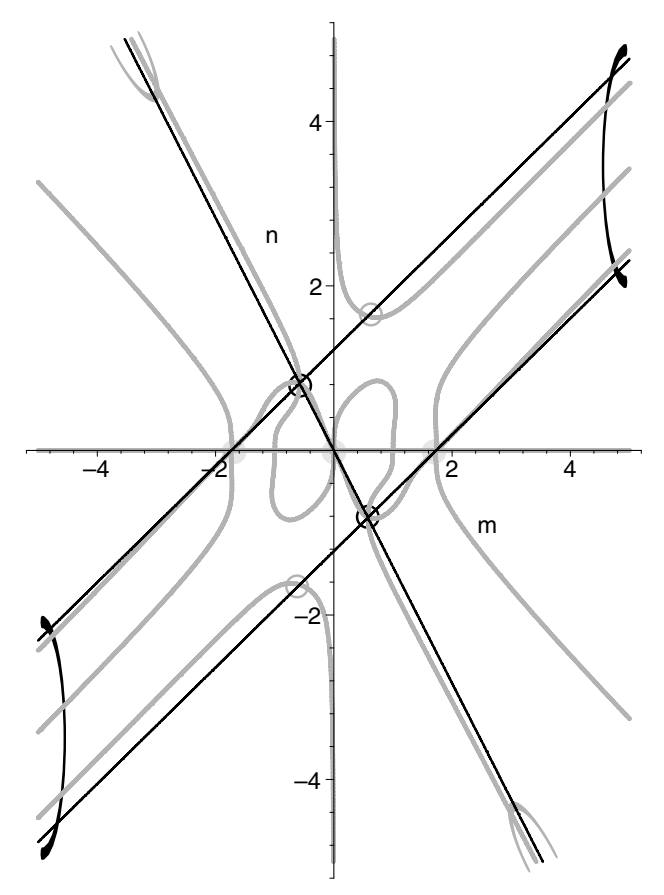

Fig. 5. Projective view of the curves defined by the homogeneous system of (11) and (12) for the regular tetrahedron.

The black curve corresponds to (12) (cocircularity) and consists of three straight lines in the case of the regular tetrahedron (as already noted in Fig. 2). The grey curve corresponds to (11) (extremality). The solutions $p_{1} p_{4}, p_{2} p_{4}$ and $p_{3} p_{4}$, circled in grey in the two figures, appear in Fig. 5 as nontangent intersections between black and grey curves (one of the three points, $p_{3} p_{4}$, is at infinity); these solutions persist in Fig. 6. The solutions $p_{1} p_{2} \times p_{4} p_{3}, p_{1} p_{4} \times p_{3} p_{2}$ and $p_{1} p_{3} \times p_{2} p_{4}$ appear circled in black in Fig. 5 at the intersections between pairs of branches of the black curve (one of the three points is at infinity). Figure 6 clearly shows that, when the tetrahedron is perturbed, each of these points splits into nontangent intersections between black and grey curves. Finally the solutions $p_{1} p_{2}, p_{1} p_{3}$ and $p_{2} p_{3}$ as tangencies of multiplicity five between black and grey curves on the axis $n=0$ are on light grey background in Fig. 5 ; in the perturbed version (Fig. 6) each of these points splits into one tangent contact between the two curves and one nontangent intersection. The situation, which is not very discernible in Fig. 6, is more clearly illustrated in Fig. 7, where the circular domain around the origin (corresponding to solution $p_{1} p_{2}$ ) is transformed by an appropriate affine transformation into an elliptical domain. This blow-up gives evidence of the existence of a tangent intersection and a simple intersection.

Theorem 9. The number of circular cylinders through four points of locally minimal (resp. maximal) radius is at most nine. 


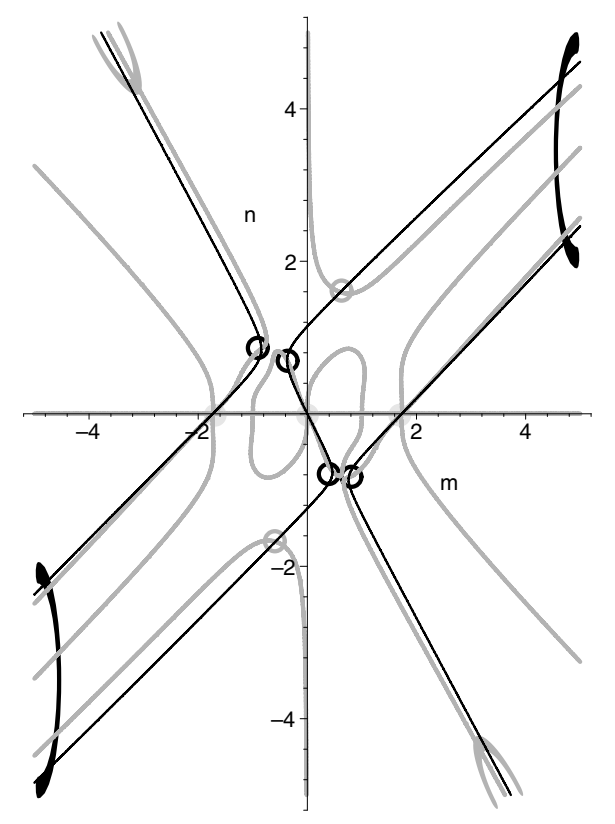

Fig. 6. Projective view of the curves defined by the homogeneous system of (11) and (12) for the perturbed regular tetrahedron.

Proof. This theorem is an easy corollary of Theorem 6. Indeed, the set of directions such that the four points are cocircular is a degree three curve of the projective space $\mathbb{P}^{2}$ (Theorem 1). This curve can be decomposed into the union of some closed curves (three projective lines in the case of the regular tetrahedron of Fig. 1). To each point of these curves there corresponds a unique value of the radius of a cylinder, and, when tracing any of these curves, we visit minima and maxima of radius in identical numbers. Since the total number of extrema, with multiplicity, is at most 18 , the total number of minima or maxima is at most nine.

Referring to the example of the (perturbed) regular tetrahedron, this bound does not appear to be tight. Indeed, we conjecture that the exact bound on the number of real maxima (or minima) is six.

\section{Appendix. Actual Computation of Solutions}

We describe two methods that we have used to solve explicitly the different problems, presented in the previous sections. The first one is a general approach based on eigencomputation, the second one is specific to curves in the plane. General references on this topic can be found in [7] and [9]. Code to solve the specific problems on cylinders we have described in this paper can be found at http://www-sop.inria.fr/galaad/demo/ Cylindre. 

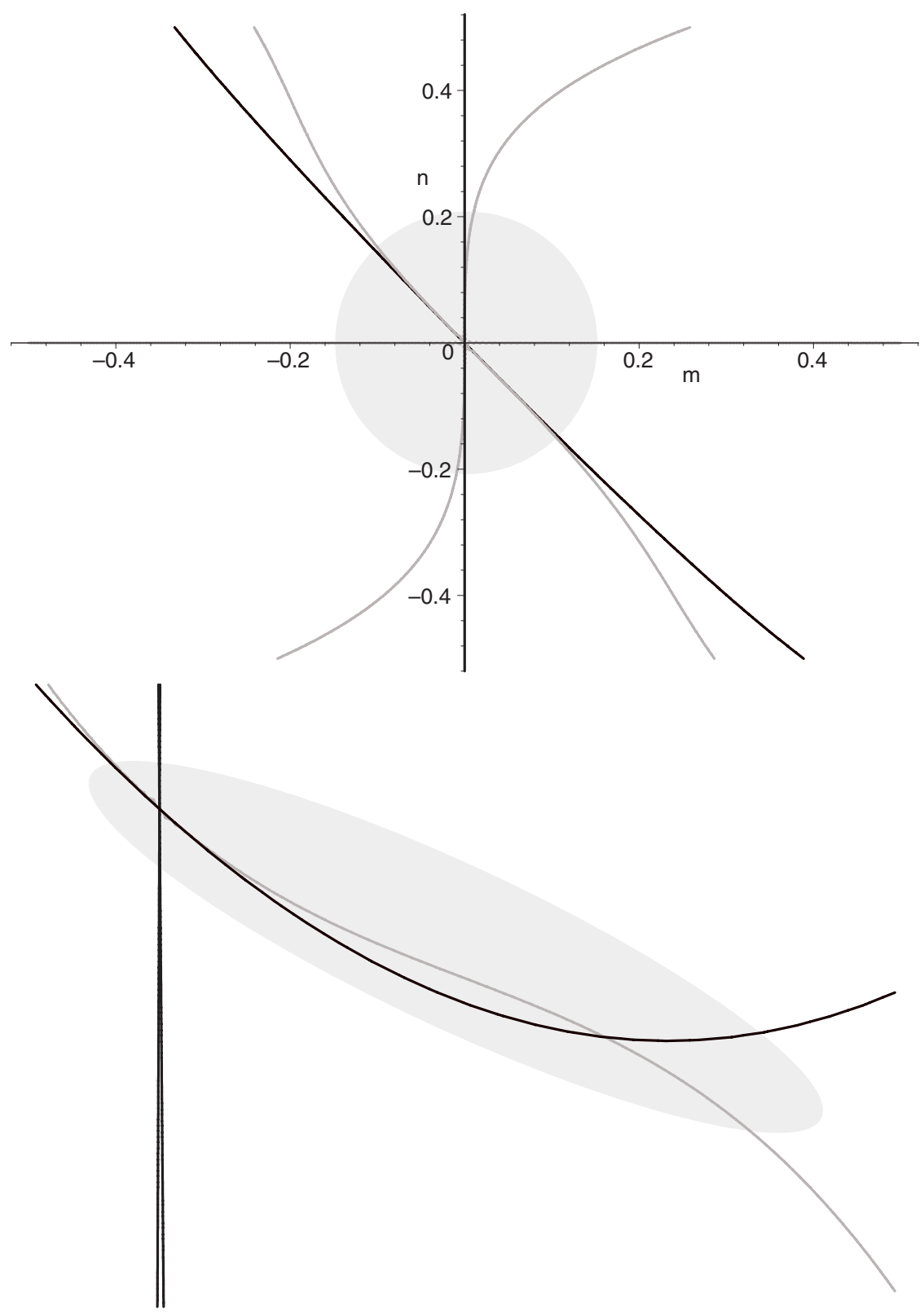

Fig. 7. Blow-up of the projective view of the solution of the homogeneous system of (11) and (12) for the perturbed regular tetrahedron in the neighborhood of the direction $p_{1} p_{2}$. 


\section{A.1. Solving Polynomial Equations by Eigencomputation}

We consider here the general setting of $m$ equations $f_{1}=0, \ldots, f_{m}=0$ in $n$ variables $t_{1}, \ldots, t_{n}$, with coefficients in $\mathbb{K}=\mathbb{R}$. The polynomials $f_{1}, \ldots, f_{m}$ are elements of the polynomial ring denoted by $R=\mathbb{K}\left[t_{1}, \ldots, t_{n}\right]$. They generate the ideal $I=\left(f_{1}, \ldots, f_{m}\right)$. In our examples, we consider two homogeneous equations $f_{1}, f_{2}$ of our problem and the equation $f_{3}=l^{2}+m^{2}+n^{2}-1$. We have $t_{1}=l, t_{2}=m, t_{3}=n$ and $R=\mathbb{R}[l, m, n]$. By considering $f_{3}$, we double the number of solutions and treat the problem in an affine setting instead of a projective one.

The quotient algebra of classes of polynomials modulo the ideal $I$ is denoted by $\mathcal{A}$. Its dual space (that is, the set of linear forms from $\mathcal{A}$ to $\mathbb{K}$ ) is denoted by $\hat{\mathcal{A}}$. Consider the map of multiplication by a variable $t_{i}$ in $\mathcal{A}$ :

$$
\begin{aligned}
M_{i}: \mathcal{A} & \rightarrow \mathcal{A} \\
a & \mapsto a t_{i} .
\end{aligned}
$$

Assume that the number of solutions our system is finite: $\zeta_{1}, \ldots, \zeta_{d}$, then $\mathcal{A}$ is a finite vector space and we have the following theorem:

Theorem 10 [13]. The common eigenvalues of all the $M_{i}$ are the $i$ th coordinates $\left(\zeta_{k}\right)_{i}$ of the roots $\zeta_{1}, \ldots, \zeta_{d}$. The common eigenvectors of all the $M_{i}^{t}$ are the evaluation operators $\mathbf{1}_{\zeta_{1}}, \ldots, \mathbf{1}_{\zeta_{m}}$ where $\mathbf{1}_{\zeta_{i}}: p \mapsto p\left(\zeta_{i}\right) \in \hat{\mathcal{A}}$.

The dimension of $\mathcal{A}$ will be the number of solutions counted with multiplicity. In our examples, it will be the Bézout bound, that is the product of the degrees of the equations. This theorem shows that solving the polynomial equations reduces to computing the matrix of the operators of multiplication $M_{i}$ and then to perform an eigenvector/eigenvalue decomposition.

The first step is performed according to the algorithm proposed in [14], and its specialization to a generic projective complete intersection [16] (which is the case that we are considering). We get matrices of size the Bézout bounds. The eigenvector computations is performed in ALP [15] with LAPACK [3] subroutines. We remove from these solutions the redundant one, corresponding to the directions $p_{1} p_{2}, p_{1} p_{3}, p_{2} p_{3}$.

The numerical computations of the solutions is performed in a $\mathrm{C}++$ implementations in ALP. Here are the timing and the accuracy that we get for the different problems:

Computations performed on an Intel PII 400128 Mo of Ram

\begin{tabular}{lcc}
\hline \multicolumn{1}{c}{ Problem } & $\begin{array}{l}\text { Time } \\
(\mathrm{sec})\end{array}$ & $\operatorname{Max}\left(\left|f_{i}\right|\right)$ \\
\hline Cylinder through five points & 0.03 & $5 \cdot 10^{-9}$ \\
Parallel cylinders through $2 \times 4$ points & 0.03 & $5 \cdot 10^{-9}$ \\
\hline Cylinder through four points, extremal radius & 2.9 & $10^{-6}$ \\
\hline
\end{tabular}


The tremendous difference of computation time between the case of Section 8 and the other two and the difference of accuracy should be noted. The explanation for these differences is in the method of resolution that we used. For the first two we already knew that the roots were of multiplicity one in the generic case, i.e., each eigenspace was of dimension one, this enabled us to compute the roots by just computing the eigenvectors of only one generic $M_{f}$. However, in the case of extremal radii the trouble was that generically the system had multiplicities and we had to consider another more sophisticated and costly method to recover the roots and their multiplicities (see for instance [6] for further details).

Figure 8 shows the six cylinders solutions for the set of points corresponding to the regular tetrahedron (the real solutions are displayed with pov-ray).

\section{A.2. Solving by Resultant Computation}

Another approach for solving a (square) polynomial systems consists in using resultant constructions. We hide a variable (that we consider as a parameter) in order to get a system with one equation more that the number of remaining variables. Then we use a resultant formulation [10] in order to get a condition on this parameter such that the overdetermined system has a solution. From this condition, we deduce the values of the hidden variable for the roots of our system. We recover the values of the other coordinates, by simple linear algebra operations.

In our example, we substitute $l$ by 1 , hide the variable $m$ and compute the Sylvester matrix of the polynomials $f_{1}, f_{2}$ with respect to the $n$. If $f_{i}$ is of degree $d_{i}$, with respect to $n$, we get a square $\left(d_{1}+d_{2}\right) \times\left(d_{1}+d_{2}\right)$ matrix $S(m)$ whose entries are polynomial in $m$

$$
S(m)=S_{k} m^{k}+\cdots+S_{1} m+S_{0},
$$

where $S_{i}$ has constant coefficients. We are looking for the values of $m$ such that the Sylvester resultant vanishes $(\operatorname{det}(\mathrm{S}(m))=0)$ or more precisely to values of $(m, n)$ such that

$$
\left[1, n, \ldots, n^{d_{1}+d_{2}-1}\right] \mathrm{S}(m)=0 .
$$

This can also be transformed into a generalized eigenproblem:

$$
\left(\left[\begin{array}{cccc}
\mathbf{0} & \mathbb{I} & \cdots & \mathbf{0} \\
\vdots & \ddots & \ddots & \vdots \\
\mathbf{0} & \cdots & \mathbf{0} & \mathbb{I} \\
S_{0}^{t} & S_{1}^{t} & \cdots & S_{k-1}^{t}
\end{array}\right]-\lambda\left[\begin{array}{cccc}
\mathbb{I} & \mathbf{0} & \cdots & \mathbf{0} \\
\mathbf{0} & \ddots & \ddots & \vdots \\
\vdots & \ddots & \mathbb{I} & \mathbf{0} \\
\mathbf{0} & \cdots & \mathbf{0} & -S_{k}^{t}
\end{array}\right]\right) \mathbf{w}=0 .
$$

The corresponding eigenvalues yield the possible values for $m$ : For each value of these values $m_{0}$, we deduce from the corresponding eigenvectors in the kernel of $S^{t}\left(m_{0}\right)$.

If the kernel is of dimension one, it is generated by $\left[1, n_{0}, \ldots, n_{0}^{d_{1}+d_{2}-1}\right]$, where $\left(1, m_{0}, n_{0}\right)$ is a solution of $f_{1}=f_{2}=0$. 


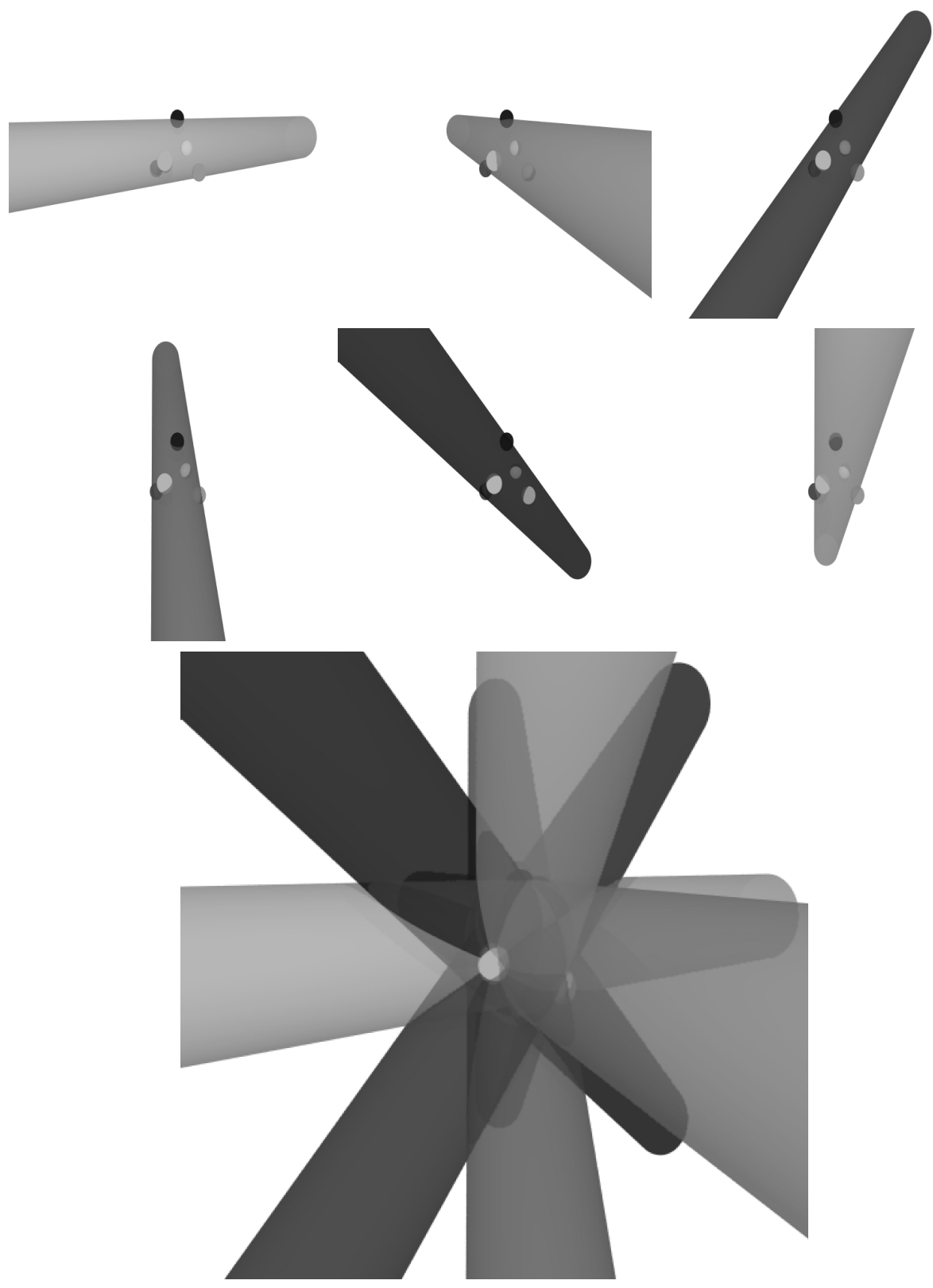

Fig. 8. The six cylinders through five points. 
If the kernel of $S^{t}\left(m_{0}\right)$ is generated by $\mathrm{K}=\left[\mathrm{k}_{1}, \ldots, \mathrm{k}_{r}\right]$ ( with $\left.S^{t}\left(m_{0}\right) \mathrm{K}=\mathbf{0}\right)$, we compute the generalized eigenvalues of $\Delta_{1}-\lambda \Delta_{0}$ where $\Delta_{0}$ (resp. $\Delta_{1}$ ) is the first $r \times r$ submatrix of $\mathrm{K}$ (resp. the $r \times r$ submatrix of $\mathrm{K}$ formed by the rows $1, \ldots, r+1$ ). From these eigenvalues, we deduce the values of the corresponding $n_{0}$ above $m_{0}$. Notice that this does not yield the multiplicity of the intersection point $\left(1, m_{0}, n_{0}\right)$ of $f_{1}=f_{2}=0$.

In order to complete our set of solutions, we also compute the points at infinity corresponding to $l=0$, which are the projective roots of the gcd of the homogeneous polynomials $f_{1}(0, m, n), f_{2}(0, m, n)$.

We refer to [10] for more information on resultant techniques for solving polynomial equations.

\section{References}

1. P.K. Agarwal, B. Aronov, and M. Sharir. Line traversals of balls and smallest enclosing cylinders in three dimensions. In Proc. 8th ACM-SIAM Sympos. Discrete Algorithms, pages 483-492, 1997.

2. P.K. Agarwal, B. Aronov, and M. Sharir. Exact and approximation algorithms for minimum-width cylindrical shells. In Proc. 11th ACM-SIAM Sympos. Discrete Algorithms, pages 510-517, 2000.

3. E. Anderson, Z. Bai, C. Bischof, J.W. Demmel, J.J. Dongara, J. Du Croz, A. Greenbaum, S. Hammerling, A. McKenney, and D. Sorensen. LAPACK: A portable linear algebra library for high-performance computers. Technical Report CS-90-105, Comput. Sci. Dept., University of Tennessee, Knoxville, 1990.

4. J. Basch, O. Devillers, M. van Kreveld, M. Teillaud, and A. Vigneron. On the number of Delaunay triangulations of the projections of a set of points in three dimensions. Rapport de Recherche, INRIA, to appear.

5. O. Bottema and G.R. VeldKamp. On the lines in space with equal distance to $n$ given points. Geom. Dedicata, 6:121-129, 1977.

6. R.M. Corless, P.M. Gianni, and B.M. Trager. A reordered Schur factorization method for zero-dimensional polynomial systems with multiple roots. In W.W. Küchlin, editor, Proc. ISSAC, pages 133-140, 1997.

7. D. Cox, J. Little, and D. O'Shea. Ideals, Varieties, and Algorithms: An Introduction to Computational Algebraic Geometry and Commutative Algebra. Undergraduate Texts in Mathematics. Springer-Verlag, New York, 1992.

8. O. Devillers and F.P. Preparata. Evaluating the cylindricity of a nominally cylindrical point set. In Proc. 11th ACM-SIAM Sympos. Discrete Algorithms, pages 518-527, 2000.

9. M. Elkadi and B. Mourrain. Géométrie algébrique effective en dimension 0. Notes de cours, Université de Nice, 2002.

10. I.Z. Emiris and B. Mourrain. Matrices in Elimination Theory. J. Symbolic Comput., 28(1\&2):3-44, 1999.

11. D. Lichtblau. Finding cylinders through 5 points in $\mathbb{R}^{3}$. Submitted.

12. I.G. Macdonald, J. Pach, and T. Theobald. Common tangents to four unit balls in $\mathbb{R}^{3}$. Discrete Comput. Geom., 26:1-17, 2001

13. B. Mourrain. Computing isolated polynomial roots by matrix methods. J. Symbolic Comput., 26(6):715738, 1998.

14. B. Mourrain. A new criterion for normal form algorithms. In M. Fossorier, H. Imai, Shu Lin, and A. Poli, editors, Proc. AAECC, volume 1719 of LNCS, pages 430-443. Springer-Verlag, Berlin, 1999.

15. B. Mourrain and H. Prieto. A framework for symbolic and numeric computations. Rapport de Recherche 4013, INRIA, 2000.

16. B. Mourrain and Ph. Trébuchet. Solving projective complete intersection faster. In C. Traverso, editor, Proc. Internat. Sympos. Symbolic and Algebraic Computation, pages 231-238. ACM Press, New York, 2000.

17. E. Schömer, J. Sellen, M. Teichmann, and C. Yap. Smallest enclosing cylinders. In Proc. 12th Annu. ACM Sympos. Computational Geometry, pages C13-C14, 1996.

Received June 8, 2001, and in revised form April 20, 2002. Online publication November 21, 2002. 